\title{
Calidad de atención de enfermería en la unidad de hemodiálisis en el área de pediatría
}

\author{
Quality of nursing care in the hemodialysis unit in the pediatric area \\ Rosa María Pérez-González ${ }^{a}$, Clara Carlson-Morales ${ }^{b}$
}

\begin{abstract}
:
Knowing the quality of hemodialysis care in the pediatric area, allows us to identify those protocols that are being carried out and identify those areas that are deficient and, on the other hand, allows us to understand the care provided in this unit. Increasing numbers of hemodialysis have patient care protocols, it is mandatory that the hemodialysis unit establish a quality accreditation system for its protocols. For this, the ISO 9001-2000 standards is one of the parameters to be used, in which it is a tool that allows us to know the processes in detail. The patient with chronic kidney disease is susceptible to receive support treatment and care in kidney patients from the diagnosis of the disease, during the different stages of treatment, for this there is a solution, I know how hemodialysis is one of the treatments with patients with diagnoses of CKD, is known as "an indispensable alternative for the treatment of advanced chronic kidney disease in children". Pediatric hemodialysis is a highly specialized treatment and should be carried out in units with specific facilities and always in a pediatric hospital under the supervision of a pediatric nephrologist (1). The purpose of this work is to know the quality of nursing care in the hemodialysis unit in the pediatrics area.
\end{abstract}

\section{Keywords:}

Hemodialysis, quality of care, Chronic Kidney Disease, ISO 9001-2000 Standard

\begin{abstract}
Resumen:
Conocer la calidad de atención en hemodiálisis en el área pediátrica, permite identificar aquellos protocolos que se están llevando a cabo e identificar aquellas áreas deficitarias y por otra parte nos permite conocer los cuidados brindado en esta unidad. Cada vez mayor número de hemodiálisis cuentan con protocolos de atención hacia los pacientes, se valora obligatoriedad que la unidad de hemodiálisis establezca sistema de acreditación de la calidad de sus protocolos. Para el ello las normas ISO 9001-2000 es uno de los parámetros a utilizar, en el cual es una herramienta que nos permite conocer los procesos en detalle. El paciente con enfermedad renal crónica es susceptible de recibir tratamiento de soporte y cuidados en pacientes renales desde el diagnóstico de la enfermedad, durante las distintas etapas de tratamiento, para ello existe una solución, sé conoce cómo hemodiálisis, es uno de los tratamientos con los pacientes con diagnósticos de la ERC, se conoce como "una alternativa indispensable para el tratamiento de la enfermedad renal crónica avanzada en niños". La hemodiálisis pediátrica es un tratamiento altamente especializado y debe llevarse a cabo en unidades con dotaciones específicas y siempre en un hospital pediátrico bajo la supervisión de un nefrólogo pediatra (1). Este trabajo tiene como propósito conocer la calidad de atención de enfermería en la unidad de hemodiálisis en el área de pediatría.
\end{abstract}

\section{Palabras Clave:}

Hemodiálisis, calidad de atención, Enfermedad Renal Crónica, Norma ISO 9001-2000

\section{Introducción}

La enfermedad renal crónico (ERC) es el resultante de diversas enfermedades crónico degenerativas, entre las que destacan la diabetes mellitus tipo y la hipertensión arterial, fenómeno que ocurre de manera similar en todo el mundo y que lamentablemente estas enfermedades conducen una trascendencia falta si es tratado. La OMS en 2015 refiere que la ERC afecta a cerca de $10 \%$ de la población mundial, según los datos de la SLANH, en América Latina unos promedios de 613 pacientes por millón de habitantes tuvieron acceso en 2011, en pacientes de hemodiálisis se sometieron 11.010, 10.921 (99\%) adultos, $5.600(51 \%)$ varones y $5.321(49 \%)$ mujeres y $89(1 \%)$ pediátricos, $64(72 \%)$ varones y $25(28 \%)$

\footnotetext{
a Rosa María Pérez González, Universidad Linda Vista AC., Pueblo Nuevo Solistahuacán, Chiapas., https://orcid.org/0000-0003-3026-2602, Email: rosa.perez@ulv.edu.mx

b Clara Carlson Morales, Universidad Linda Vista AC., Pueblo Nuevo Solistahuacán, Chiapas., https://orcid.org/0000-0002-9752-8033, 
mujeres (2). ERC muestra uno de los factores principales de la salud pública (3).

La hemodiálisis representa uno de los factores problemáticas en el área de salud debido a los altos índices de la insuficienciarenal, como consecuencia presentaun alto gasto económico, tanto en morbimortalidad; actualmente las enfermedades crónicas producen el $60 \%$ de los 58 millones de muertes en el mundo cada año y representa alto carga económica para las sociedades, quiere decir que casi el $80 \%$ de los recurso sanitarios de los países desarrollados son consumidos por la necesidad de los pacientes con enfermedades crónicas (3). Se menciona que tan solo un $40 \%$ de los pacientes incidentes en nuestro medio reciben como primera modalidad de tratamiento sustitutivo y si bien la diálisis peritoneal, el $9 \%$ de los pacientes pediátricos reciben tratamiento de hemodiálisis, el $4 \%$ con diálisis peritoneal y afortunadamente el $87 \%$ con un trasplante renal (4).

Las expectativas de la tasa del crecimiento en México son preocupantes en los próximos años. Será un reto que enfrentará al sistema de salud del país, entre muchos aspectos, para ello incluye especialmente la disposición del personal de sanitario calificado para la calidez atención sobre este grupo (5). En términos epidemiológicos, el resultado final de todoelloes doble: por un lado, el riesgo de desarrollo de insuficiencia renal es más elevado en los sujetos con una enfermedad vascular (6). Por lo tanto, este trabajo tiene como propósito conocer la calidad de atención de enfermería en la unidad de hemodiálisis en el área de pediatría.

\section{Desarrollo}

Conceptos:

Calidad: "Para la Norma ISO 9001-2000 es el conjunto de características de una entidad que le confieren la aptitud para satisfacer las necesidades, tanto establecidas como las implícitas"(7).

Sistema de calidad: "Es la estructura organizativa, procedimientos, procesos y recueros necesarios que permite implantar una gestión de calidad y ha de ser todo lo amplio que se necesario para alcanzar los objetivos de calidad" (7).

La Enfermedad Renal Crónica (ERC): Muestra uno de los factores principales de la salud pública la ERC, de igual manera por su importante morbimortalidad cardiovascular, sumando los costes sociales y económicas que requiere. Para ello requiere se plantea varios motivos para el manejo integral de los pacientes con ERC:

a) la existencia de múltiples comodidades en esta población requiere una mejor coordinación de la atención médica y planes específicos para algunas patologías, especialmente la diabetes mellitus tipo 2 y las enfermedades cardiovasculares, b) altas tasas de ingresos hospitalarios y morbilidad, que se podría reducir con un buen manejo del control ambulatorio y principalmente fomentar la prevención a través de la medicación,

c) la existencia de varios procesos que ha resultado mayor mejoría de los resultados clínicos y

d) una atención elevada de la población con ERC (3). Los procesos y complicaciones mencionados con la ERC existe un tratamiento por la cual los pacientes acuden para mejor su condición física, actualmente se conoce como Hemodiálisis se conoce como "una alternativa indispensable para el tratamiento de la enfermedad renal crónica avanzada en niño" (1), o bien es un procedimiento que se realiza en la Unidad de hemodiálisis, como principal función constituye la depuración extra-renal es un tratamiento eficaz para la enfermedad renal crónica avanzada en niños, sin embargo existe otro tratamiento agudo se conoce como la diálisis peritoneal consiste en la eliminación de tóxicos y fármacos de forma eficaz, es la regulación rápido del medio interno o un alivio sintomático del edema o de la sobrecarga hídrico asociada (4). Estos tipos de tratamiento se asocia por problemas físicos como "alteraciones del crecimiento, nutrición y desarrollo; con alteraciones de óseas y vasculares" con una disminución de la calidad de vida relacionado con los procedimientos en la unidad y en el área hospitalaria, de igual manera de la separación de los familiares y últimamente las limitaciones escolares, sensación de pérdida de independencia y de la identidad e integridad corporal (4). La diabetes mellitus y la enfermedad coronaria son comorbilidad prevalente en pacientes en diálisis. Según la encuesta anual 2007 de la Sociedad Chilena de Nefrología 33,8\% de los pacientes en programa de hemodiálisis tiene diagnóstico de diabetes y de ellos 64,6\% tiene actividad limitada o incapacidad de autocuidado (8).

Aspectos psicológicos: Durante cada procedimiento llevado a cabo en la unidad de la hemodiálisis los pacientes manifiestan aspectos psicológicos en la vida de cada uno de ellos, además los familiares serán afectados en esta cuestión. Como primer trastorno presenta la depresión de alta prevalencia con un 10-66\% de los pacientes. Asimismo los trastornos de ansiedad es uno de los factores presentes dentro de las experiencias del enfermo renal se localiza en las fases finales de la enfermedad si no se trata de manera preventiva; la tasa de la prevalencia del trastorno de ansiedad oscila entre $45,7 \%$ de manera negativamente afecta la calidad de vida al paciente (9).

Calidad de atención: La gestión de calidad es disminuir la variabilidad de la práctica clínica, tanto en el momento de 
establecer una indicación. La implantación de este tipo de sistema de gestión de la calidad se basa en la normalización y control de los procesos que se realizan con el fin de que los resultados cumplan con los estándares de calidad fijos (10).

En la unidad de hemodiálisis según la norma ISO-9001-2000 hace mención un protocolo para esta unidad, este lineamiento sirve para evaluarla calidad de atención brindadoen cada uno de los pacientes durante los procedimientos. En la tabla 1 se muestra la lista de protocolo para el manejo en esta unidad y para la realización de los procedimientos.

Tabla 1.

Protocolo del sistema de calidad para hemodiálisis
\#. Procedimiento
1. Recepción del paciente en hemodiálisis.
2. Montaje y cebado del circuito de HD.
3. Conexión.
4. Programación de la HD.
5. Cuidados de enfermería en la sesión de HD.
6. Extracción de analíticas.
7. Incidencias técnicas en HD.
8. Incidencias médicas en hemodiálisis.
9. Desconexión.
10. Desinfección y limpieza de monitores.
11. Vigilancia y cuidado de accesos vasculares.
12. Vacunación de la hepatitis B.
13. Medidas de aislamiento y protección.
14. Reanimación cardiopulmonar.
15. Recepción de pacientes desplazados.
16. Colocación catéter temporal.
17. Cuidados catéteres permanentes y temporales.
18. Solicitud y recogida de medicación al hospital.
19. Limpieza diaria.
20. Gestión de residuos biosanitarios específicos.

21. Control de monitores de HD y de la planta de aguas.

22. Control de las analíticas y exploraciones complementarias.

23. Seguimiento médico del paciente de HD.

24. HD de pacientes agudos

Nota: Recuperado de Aseguramiento de la calidad y certificación de una unidad de hemodiálisis, de Arenas, Gil TM, Egea J., Sirvent A. E, Giménez A. 2003; revistanefrologia.com. 1(30):38-46. (7)

En un estudio realizado con el personal del área de la salud referente a la calidad de atención, se apreciaron diferencias en las percepciones diferencias en las percepciones entre medicina y enfermería. El personal de medicina laboralmente se sintieron más satisfechos con sus superiores y percibieron a sus unidades como más eficaces, en cambio, el personal de enfermería percibió mayor flexibilidad en sus horas de trabajo, presentan un mayor cantidad de estrés y una mejor atención del personal (5).

Existe otro factor relacionado por la falta de atención brindado por el personal, se ha realizado estudiados ampliamente se conoce como el síndrome de Burnout, se define como: "síndrome de agotamiento emocional, despersonalización y baja realización personal que puede aparecer un individuo cuyo trabajo implica atención o ayuda a las personas", se caracteriza por la falta de empatía del personal y aptitudes de irritabilidad y desmotivación por otro lado esta profesión necesita mucha entrega y sobre todo empatía hacia los pacientes, lo primordial en este momento es una atención de calidad. En un estudio realizado demostró tres características de bourner entre los que se encuentran: trastorno psicosomático, emocionales y conductuales. Este tipo de trabajo que emplean las enfermeras en el área de hemodiálisis trabaja con los aspectos emocionales, la enfermedad, la muerte, la angustia y la muerte (11).

\section{Conclusión}

Se puede concluir que con los respaldos en las investigaciones presentadas puede mejor la calidad de atención hacia los pacientes; a veces pasa desapercibido el gran aporte del sistema de calidad de enfermería, impactando o afectando el trabajo del personal de salud. Por lo que es importante recordar la gran importancia de refrescar o conocer la atención de los pacientes en el área de hemodiálisis, ya que nos permite tener una perspectiva amplia y es así que invito a todo el personal del área de la salud y en especial al personal administrativo y de enfermería en la unidad de hemodiálisis para actualizarse y cumplir con los protocolos establecidos para brindar una calidad de atención en el área de pediatría.

\section{Referencias}

1. Alonso M. A, López-Viota JF. Hemodiálisis pediátrica. Protoc diagn ter pediat. 2014;(1):403-20.

2. Méndez-Durán A, Méndez-Bueno F, Tapia-Yáñez T, Muñoz-Montes A, Aguilar-Sánchez L. Diálisis y Trasplante. Elsevier [Internet]. 2010;31(1):7-11. Disponible en: https://www.elsevier.es/es-revistadialisis-tra splante-275-pdf-S1886284510700047

3. Ramos R, Molina M. Nuevos modelos de gestión de asistencia integral en nefrología. Rev Nefrol. 2013;33(3):301-7.

4. Melgar AA, Lopez PO. Hemodiálisis Pediátrica. 2019; 
5. Russell MR, Gómez LLT, Domínguez RLP, Santiago RE, Cervantes ML. El clima laboral en las unidades de hemodiálisis en México . Rev Nefrol. 2011;31(1):76-83.

6. Marín R, Goicoechea MA, Gorostidi M, Cases A, Díez J, Escolar G, et al. Guía de la Sociedad Española de Nefrología sobre riñón y enfermedad cardiovascular. Rev Nefrol. 2006;26(1):31-44.

7. Arenas, Gil TM, Egea J. ., Sirvent A. E, Giménez A. Aseguramiento de la calidad y certificación de una unidad de hemodiálisis según normas ISO-9001-2000. Nefrologia [Internet]. 2003;1(30):38-46. Disponible en: https://www.revistanefrologia.com/es-pdf-X0211699503015654

8. Carlos-Zúñiga S, Juan-Dapueto $P$, Hans-Müller O, Lilian-Kirsten L, Raquel-Alid A, Liliana-Ortiz M. Evaluación de la calidad de vida en pacientes en hemodiálisis crónica mediante el cuestionario "Kidney Disease Quality of Life (KDQOL-36)" Carlos. Rev Med Chile. $2009 ; 137: 200-7$.

9. Leiva-santos JP, Sánchez-hernández R, García-llana H, Fernándezreyes MJ, Heras-benito M, Molina-ordas Á, et al. Cuidados de soporte renaly cuidados pa liativos renales : revisión y propuesta en terapia renal sustitutiva. Rev Nefrol. 2012;32(1):20-1.

10. Arenas MD, Lorenzo S, Angoso M, Aranaz J. Calidad en las unida des de nefrología españolas. Rev Nefrol. 2006;26(2):234-45.

11. Mariscal LM, García BM, Centeno BR. El síndrome de Burnout y el nivel de empatía en el enfermero de diálisis. Enferm Nefrol. 2017;20(1):63-7. 